\title{
Remote Cerebellar Hemorrhage Following Lumboperitoneal Shunt Insertion: A Rare Case Report
}

\author{
Fatih Ayvalık ${ }^{1}$ Rafet Ozay ${ }^{1} \quad$ Erhan Turkoglu ${ }^{1} \quad$ Mehmet Serdar Balkan ${ }^{1}$ Zeki Şekerci ${ }^{1}$ \\ ${ }^{1}$ Clinic of Neurosurgery, Ministry of Health Dışkapı Yıldırım Beyazıt \\ Training and Research Hospital, Ankara, Turkey \\ Surg J 2016;2:e139-e142 \\ Address for correspondence Mehmet Serdar Balkan, MD, Clinic of \\ Neurosurgery, Ministry of Health Dışkapı Yıldırım Beyazıt Training and \\ Research Hospital, İrfan Baştuğ Cad. Dışkapı-Altındağ, Ankara 06110, \\ Turkey (e-mail: mserdarbalkan@hotmail.com).
}

\author{
Abstract \\ Keywords \\ - cerebellar \\ hemorrhage \\ - complication \\ - lumboperitoneal \\ shunt
}

Idiopathic intracranial hypertension is characterized by high intracranial pressure without hydrocephalus or intracranial mass. Surgical treatment includes optic nerve fenestration and insertion of ventriculoperitoneal and lumboperitoneal (LP) shunts. For decreasing intracranial pressure, cerebrospinal fluid (CSF) LP shunt is widely used for the surgical management; it also carries complications such as shunt migration, venous sinus thrombosis, subarachnoid hemorrhage, and subdural and intracerebral hematoma. A 52-year-old man was admitted to the neurosurgery clinic with severe headache, retro-orbital pain, and blurred vision. Lumbar puncture demonstrated that the CSF opening pressure was $32 \mathrm{~cm} \mathrm{H}_{2} \mathrm{O}$. A nonprogrammable LP shunt with two distal slit valves was inserted. Shortly after the surgery, his condition deteriorated and he became comatose. Immediate computed tomography scan revealed cerebellar hemorrhage and acute hydrocephalus. Development of remote cerebellar hemorrhage following LP shunt is rare. We discuss this rare event and the applicable literature.

Idiopathic intracranial hypertension (IIH) is characterized by high intracranial pressure without hydrocephalus or intracranial mass. ${ }^{1,2}$ The incidence is 1 to $3 / 100,000$ in the general population and $21 / 100,000$ in the obese population. ${ }^{3}$ The pathophysiological causes of rising intracranial pressure are still unknown. One of the theories is decreased absorption due to increased cerebral venous pressure. ${ }^{1}$ The presence of transverse sinus stenosis is radiologically shown in a large number of patients with IIH. However, it is still not clear whether this finding increases intracranial pressure. ${ }^{4}$

The most common symptoms were headache $(92 \%)$ and blurred vision (35 to 60\%). Approximately $67 \%$ of these patients had headaches refractory to medical treatment, and progressive optic neuropathy was identified in $52 \%$ of the patients. ${ }^{2,5}$ Treatment of the IIH depends on the variety of the symptoms and associated clinical findings. If the patient only has headache, medical treatment including diuretics (acetazolamide, topiramate) and analgesics is appropriate.

received

June 15, 2016 accepted after revision October 13, 2016
If the patient has headache with accompanying loss of the vision, surgical treatment including optic nerve fenestration and placement of ventriculoperitoneal (VP) and lumboperitoneal (LP) shunts should be more appropriate. ${ }^{2,6,7}$ Rarely, steroids are recommended in cases of radiologic transverse sinus stenosis and obesity until surgical treatment can be applied. ${ }^{4,5,8}$

For decreasing intracranial pressure, cerebrospinal fluid (CSF) LP shunt is widely used for the surgical management, although it carries complications such as shunt migration, venous sinus thrombosis, subarachnoid hemorrhage, and subdural and intracerebral hematoma. ${ }^{9-13}$ We present a case of cerebellar hemorrhage after LP shunt insertion, which resulted in rapid deterioration and coma shortly after surgery. Development of remote cerebellar hemorrhage following LP shunt is a rare complication. We also provide a presumptive biomechanism of intracerebral hematoma due to LP shunt insertion.
Copyright $\odot 2016$ by Thieme Medical Publishers, Inc., 333 Seventh Avenue, New York, NY 10001, USA. Tel: +1(212) 584-4662.
License terms

10.1055/s-0036-1594245. ISSN 2378-5128.

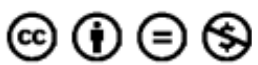




\section{Case Report}

A 52-year-old man was admitted to the neurosurgery clinic with severe headache, retro-orbital pain, and blurred vision. His headache got worse over the next 3 days. He did not have a remarkable history of using any drugs such as steroids, tetracycline, nitrofurantoin, or vitamins. His physical examination was normal, and his body mass index was 40 . Neurologic examination was normal but ophthalmologic examination by fundoscopy revealed bilateral papilledema with tortuous vessels. Ophthalmology consultation ruled out other causes of papilledema. Lumbar puncture demonstrated that the CSF opening pressure was $32 \mathrm{~cm} \mathrm{H}_{2} \mathrm{O}$. Laboratory evaluations of blood and CSF were within normal limits. Brain magnetic resonance imaging (MRI) and magnetic resonance venography revealed normal parenchymal and orbital findings and no dural sinus thrombosis. He was started on acetazolamide ( $3 \times 250 \mathrm{mg}$, orally) and furosemide $(2 \times 40$ $\mathrm{mg}$, orally) for 4 weeks. However, his symptoms partially improved with serial lumbar punctures. Therefore, a nonprogrammable LP shunt with two distal slit valves (Phoenix Biomedical Corp., Valley Forge, Pennsylvania, United States) was inserted. There was no perioperative complication, and the patient was mobilized 24 hours after surgery. Shortly after the mobilization, his condition deteriorated and he became comatose. Glasgow Coma Scale score was 6/15. Immediate computed tomography scan revealed a remote cerebellar hemorrhage and acute hydrocephalus due to fourth ventricular compression ( $\mathbf{- F i g . ~ 1 )}$ ). The patient was intubated and transferred to the intensive care unit. The LP shunt was obliterated and external ventricular drainage was inserted for hydrocephalus. Cranial MRI and magnetic resonance angiography revealed no aneurysm or arteriovenous malformation as a cause of cerebellar hemorrhage (- Fig. 2). He died 9 days after the second surgery.

\section{Discussion}

$\mathrm{IIH}$ is characterized by increased intracranial pressure without hydrocephalus or mass lesion and with normal CSF composition. The maximum available medical treatment, such as steroids, acetazolamide, and serial lumbar punctures, should be administered as first-line therapy before placing a shunt. ${ }^{2,7}$ Rosenberg et al reported that complications are common and limit the effectiveness of all kind of shunts in procedures for IIH. In their series, 37 patients had a total of 73 LP shunts and 9 VP shunts. ${ }^{9,10}$ Only 14 patients remained "cured" after a single surgical procedure. The average time between shunt insertion and shunt replacement was 9 months, although $64 \%$ of shunts needed revision within 6 months. Shunt failure (55\%) and headaches due to intracranial hypotension (21\%) were the most common causes for revision. ${ }^{10} \mathrm{LP}$ shunts are also associated with a lower complication rate than VP shunts in the treatment of IIH. ${ }^{11}$

Although placement of an LP shunt is an effective and relatively safe procedure, a variety of complications associated with LP shunt have been reported in the literature; these include malfunction requiring revision, infection, and migration or fracture of catheter. Some rare complications have also been reported, including radiculopathy, foraminal migration of shunt, tonsillar herniation, spinal deformities, subarachnoid hemorrhage, and acute subdural hematoma. ${ }^{9-12}$ Development of intracerebral hemorrhage following LP shunt is a quite rare complication, and only two cases have been reported in the literature. ${ }^{10,13}$ As stated by Turkoglu et al, ${ }^{11}$ the potential pathogenesis is overdrainage, but lack of any subdural effusion or blood argues against this possibility. Lumbar CSF drainage may result in a reduction of CSF volume with a related lowering in intracranial pressure. This may eventually increase the transmural pressure gradient of the vessels, leading to a secondary wall stress rupture.

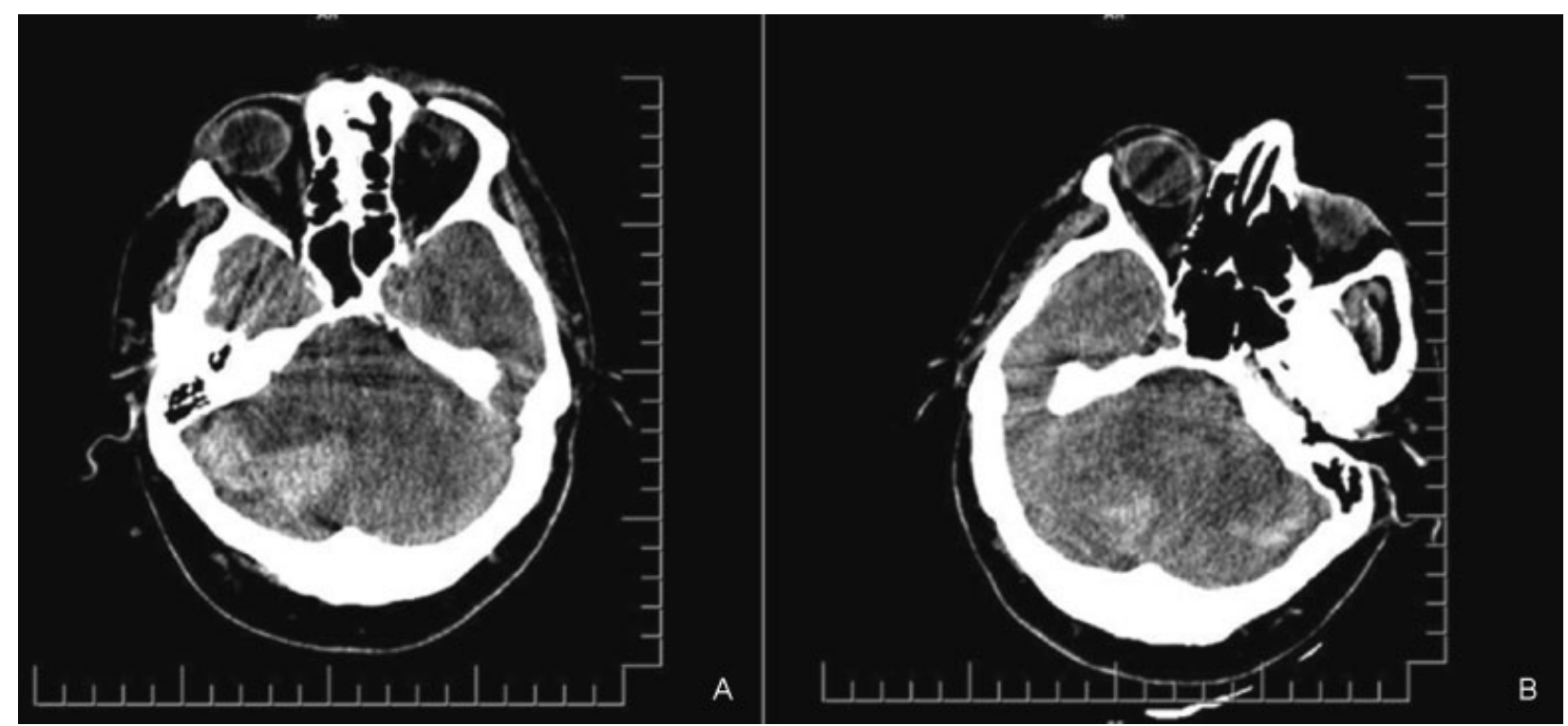

Fig. 1 Postoperative cranial noncontrast computed tomography axial section. (A) Hyperdense lesion showing right cerebellar hemorrhage with moderate mass effect. (B) Bilateral cerebellar hemorrhage. 


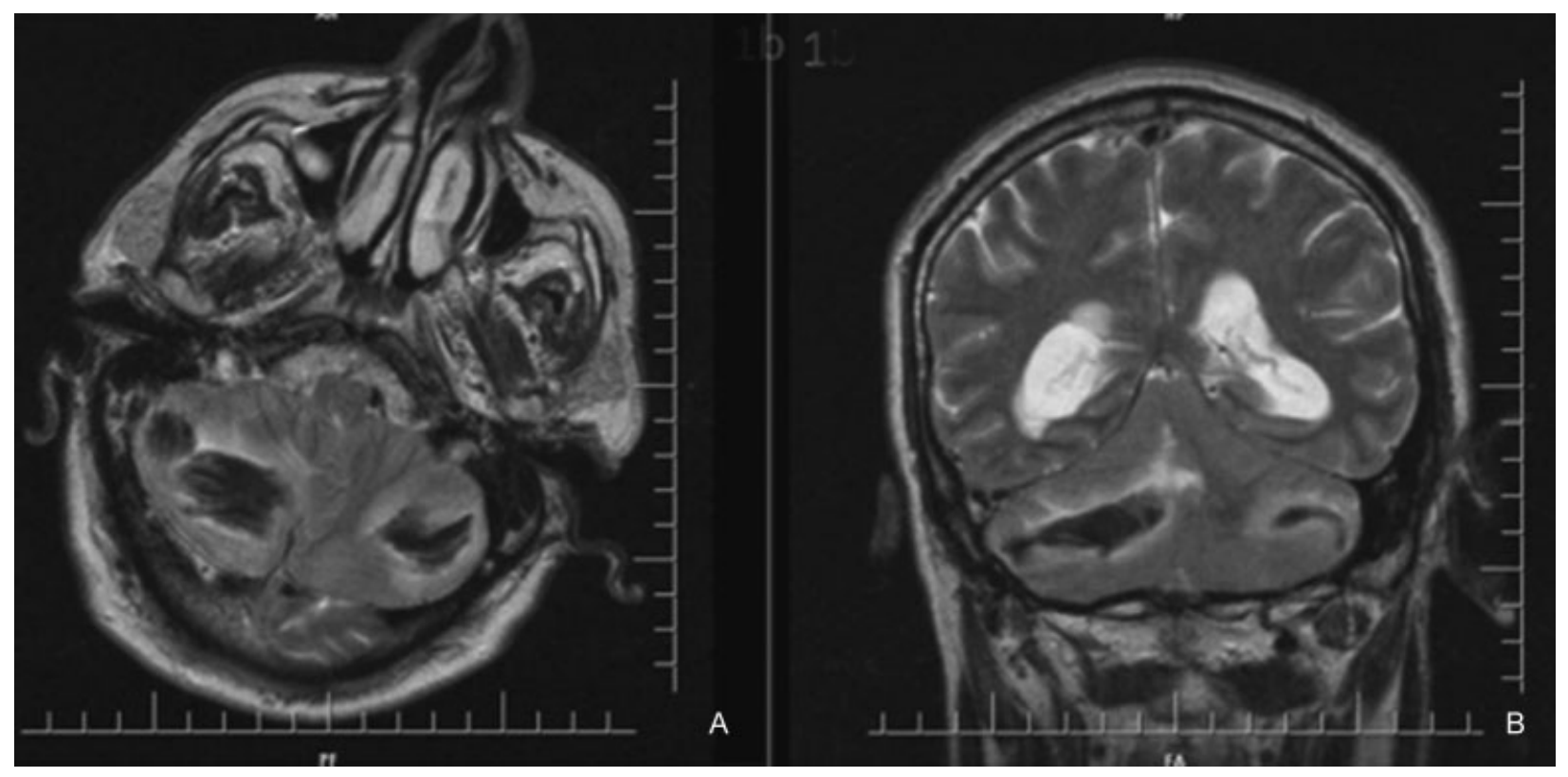

Fig. 2 Postoperative T2-weighted cranial magnetic resonance imaging. (A) Axial section and (B) coronal section showing bilateral cerebellar hemorrhage with fourth ventricle compression.

Overdrainage of the CSF during the operation may lead to intracerebral hemorrhage. However, cerebellar hemorrhage associated with LP shunt insertion has not been reported in the English language literature. Cerebellar hemorrhage mostly develops remotely from the surgical site and especially occurs after spinal surgeries and supratentorial transcranial surgery due to intracranial hypotension related to a high amount of CSF drainage. ${ }^{14,15}$ Drainage of CSF stretches the bridging veins in the posterior fossa, causing temporary occlusion, and increases intraparenchymal venous pressure, which mostly causes venous hemorrhage. ${ }^{14-16}$ The zebra sign was identified by Brockmann et al as a bleeding pattern of cerebellum following CSF loss. ${ }^{17}$ However, in our case blood had already accumulated homogenously in the posterior fossa at the time of brain imaging (-Fig. 1).

The LP shunt used in our case was not a programmable shunt. However, it had a slit valve at the distal end, and excessive drainage of CSF did not occur during the operation. After 24 hours, followed by mobilizing the patient, neurologic deterioration and sudden unconsciousness occurred. These findings suggest that an LP shunt could cause excessive CSF drainage depending on the position. Lumbar CSF drainage reduces CSF volume with a related lowering in intracranial pressure. This may eventually increase traction in the posterior fossa bridging veins and may cause remote cerebellar hemorrhage.

In conclusion, although remote cerebellar hemorrhage following an LP shunt procedure is not common, this complication should be remembered when neurologic deterioration occurs after LP shunt placement.

\section{References}

1 Biousse V, Bruce BB, Newman NJ. Update on the pathophysiology and management of idiopathic intracranial hypertension. J Neurol Neurosurg Psychiatry 2012;83(5):488-494
2 Brazis PW. Clinical review: the surgical treatment of idiopathic pseudotumour cerebri (idiopathic intracranial hypertension). Cephalalgia 2008;28(12):1361-1373

3 Durcan FJ, Corbett JJ, Wall M. The incidence of pseudotumor cerebri. Population studies in Iowa and Louisiana. Arch Neurol 1988;45(8):875-877

4 Dykhuizen MJ, Hall J. Cerebral venous sinus system and stenting in pseudotumor cerebri. Curr Opin Ophthalmol 2011;22(6): 458-462

5 Wall M, George D. Visual loss in pseudotumor cerebri. Incidence and defects related to visual field strategy. Arch Neurol 1987; 44(2):170-175

6 Corbett JJ, Nerad JA, Tse DT, Anderson RL. Results of optic nerve sheath fenestration for pseudotumor cerebri. The lateral orbitotomy approach. Arch Ophthalmol 1988;106(10): 1391-1397

7 Celebisoy N, Gökçay F, Sirin H, Akyürekli O. Treatment of idiopathic intracranial hypertension: topiramate vs acetazolamide, an openlabel study. Acta Neurol Scand 2007;116(5):322-327

8 Thambisetty M, Lavin PJ, Newman NJ, Biousse V. Fulminant idiopathic intracranial hypertension. Neurology 2007;68(3): 229-232

9 Riffaud L, Moughty C, Henaux PL, Haegelen C, Morandi X. Acquired Chiari I malformation and syringomyelia after valveless lumboperitoneal shunt in infancy. Pediatr Neurosurg 2008;44(3): 229-233

10 Rosenberg ML, Corbett JJ, Smith C, et al. Cerebrospinal fluid diversion procedures in pseudotumor cerebri. Neurology 1993; 43(6):1071-1072

11 Turkoglu E, Kazanci B, Karavelioglu E, Sanli M, Kazanci B, Sekerci Z. Intracerebral hematoma following lumboperitoneal shunt insertion: a rare case report. Turk Neurosurg 2011;21(1):94-96

12 Duthel R, Nuti C, Motuo-Fotso MJ, Beauchesne P, Brunon J. [Complications of lumboperitoneal shunts. A retrospective study of a series of 195 patients (214 procedures)]. Neurochirurgie 1996; 42(2):83-89, discussion 89-90

13 Suri A, Pandey P, Mehta VS. Subarachnoid hemorrhage and intracereebral hematoma following lumboperitoneal shunt for pseudotumor cerebri: a rare complication. Neurol India 2002;50(4): 508-510 
e142 Remote Cerebellar Hemorrhage Following LP Shunt Insertion Ayvalık et al.

14 Friedman JA, Ecker RD, Piepgras DG, Duke DA. Cerebellar hemorrhage after spinal surgery: report of two cases and literature review. Neurosurgery 2002;50(6):1361-1363, discussion 1363-1364

15 Friedman JA, Piepgras DG, Duke DA, et al. Remote cerebellar hemorrhage after supratentorial surgery. Neurosurgery 2001; 49(6):1327-1340
16 Bloch J, Regli L. Brain stem and cerebellar dysfunction after lumbar spinal fluid drainage: case report. J Neurol Neurosurg Psychiatry 2003;74(7):992-994

17 Brockmann MA, Nowak G, Reusche E, Russlies M, Petersen D. Zebra sign: cerebellar bleeding pattern characteristic of cerebrospinal fluid loss. Case report. J Neurosurg 2005;102(6):1159-1162 